\title{
The Metacognitions about Smoking Questionnaire: Development and psychometric properties
}

\author{
Submission for the Special Issue \\ 'Contemporary Perspectives on Cognition in Addictive Behaviors' \\ Word count (all sections included): 5,781 \\ Date of $1^{\text {st }}$ submission: $10 / 10 / 2014$ \\ Date of $2^{\text {nd }}$ submission: 25/10/2014
}

\begin{abstract}
Ana V. Nikčević ${ }^{\mathrm{a},{ }^{*}}$, Gabriele Caselli ${ }^{\mathrm{b}, \mathrm{d}}$, Adrian Wells ${ }^{\mathrm{c}}$ and Marcantonio M. Spada ${ }^{\mathrm{d}}$
${ }^{a}$ Department of Psychology, Kingston University, Kingston upon Thames, UK

${ }^{\mathrm{b}}$ Studi Cognitivi, Modena, Italy

${ }^{\mathrm{c}}$ Division of Clinical Psychology, The University of Manchester, Manchester, UK, Manchester Mental Health and Social Care NHS Trust, and Dept. of Psychology NTNU, Trondheim, Norway.

${ }^{\mathrm{d}}$ Department of Psychology, School of Applied Science, London South Bank University, London, UK

* Correspondence to: School of Psychology, Criminology and Sociology, Faculty of Arts and Social Sciences, Kingston University, Kingston upon Thames, United Kingdom. Tel. +44 (0)20 8417 9000, e-mail a.nikcevic@kingston.ac.uk.
\end{abstract}

(C) 2016, Elsevier. Licensed under the Creative Commons Attribution-NonCommercialNoDerivatives 4.0 International http://creativecommons.org/licenses/by-nc-nd/4.0/ 


\begin{abstract}
Objectives: Recent research has suggested that metacognitions may play a role in smoking. The goal of the current set of studies was to develop the first self-report instrument of metacognitions about smoking. Method: We conducted three studies with samples of smokers ( $n=222, n=143, n=25)$ to test the structure and psychometric properties of the Metacognitions about Smoking Questionnaire and examined its capacity to predict smoking behaviour. Results: Exploratory and confirmatory factor analyses supported a four-factor solution: positive metacognitions about cognitive regulation, positive metacognitions about emotional regulation, negative metacognitions about uncontrollability, and negative metacognitions about cognitive interference. Internal consistency, predictive and divergent validity, and temporal stability were acceptable. The metacognitions factors correlated positively with daily cigarette use and levels of nicotine dependence, and they contributed to the prediction of these outcomes over and above smoking outcome expectancies. Conclusions: The Metacognitions about Smoking Questionnaire was shown to possess good psychometric properties, as well as predictive and divergent validity within the populations that were tested. The metacognitions factors explained incremental variance in smoking behaviour above smoking outcome expectancies.
\end{abstract}


Key words: metacognition; metacognitions about smoking; nicotine use; psychometric measure; smoking; smoking outcome expectancies.

\section{Introduction}

In the metacognitive model of psychopathology Wells and Matthews (1994; 1996) advanced the idea that psychological dysfunction is linked to maladaptive metacognitions. Metacognitions refer to knowledge or beliefs about one's own cognitive system and factors that affect its functioning and regulation (Wells, 1995). According to Wells (2000) metacognitions in the knowledge domain can be usefully divided into two broad sets: (1) positive metacognitions about control strategies that impact on inner events such as "Rumination will help me get things sorted out in my mind" or "If I worry I will be prepared"; and (2) negative metacognitions concerning the significance, controllability and danger of inner events, such as "It is bad to have certain thoughts" or "I cannot stop ruminating".

Metacognitions have been found to be associated with a wide array of psychological and behavioural problems (for a full review see Wells, 2009; 2013) including addictive behaviours such as alcohol use (e.g. Clark et al., 2012; Spada, Caselli \& Wells, 2009; Spada \& Wells, 2005; 2006; 2008; 2009; 2010), gambling (e.g. Lindberg, Fernie \& Spada, 2011; Spada, Giustina, Rolandi, Fernie \& Caselli, 2014), and problematic Internet use (Spada, Langston, Nikčević \& Moneta, 2008). Within the area of smoking, two preliminary studies (Nikčević \& Spada, 2008; Spada, Nikčević, Moneta \& Wells, 2007) have found evidence of: (1) a positive association between negative metacognitions (lack of cognitive confidence and beliefs about the need to control thoughts) and nicotine use that is independent of negative emotions; and (2) an independent contribution (over negative emotions) of beliefs about the need to control thoughts towards category membership as a nicotine dependent smoker.

Further research undertaken by Nikčević \& Spada (2010) has identified the existence of specific positive and negative metacognitions about smoking. Positive metacognitions about smoking have been conceptualised as a specific form of outcome expectancy likely to play a central 
role in motivating individuals to engage in smoking as a means of cognitive-emotional regulation. Examples of positive metacognitions about smoking include: "Smoking helps me to think things through" or "Smoking helps me to feel less pressure”. Negative metacognitions about smoking have been conceptualised as beliefs concerning the uncontrollability of smoking and smoking-related thoughts (e.g. "I cannot stop thinking about cigarettes”), and the perceived negative impact of smoking on self-appraisal and cognitive functioning (e.g. "Smoking is a sign of my low will power"). These metacognitions are thought to play a crucial role in the perpetuation of smoking by becoming activated during and following a smoking episode, and triggering negative emotional states that compel a person to use more (Nikčević \& Spada, 2010; Spada, Caselli \& Wells, 2013).

The current study builds on these findings by presenting the development and preliminary validation of a self-report instrument designed to assess metacognitions in smoking. We hypothesized that this newly developed instrument would have a significant association with daily cigarette use and severity of nicotine dependence and that this relationship would be maintained when controlling for smoking outcome expectancies, a related but separate construct to meacognitions. The development of this self-report instrument may facilitate further quantitative research investigating the role of specific metacognitions involved in the activation, perseveration and escalation of smoking. It may also provide a first tool to identify individuals with this type of metacognitive profile.

\section{Study 1: Construction of Metacognitions about Smoking Questionnaire (MSQ)}

\subsection{Method}

\subsubsection{Participants}

A sample of 222 individuals (131 female) agreed to participate in the study which was approved by the ethics committee of a London (United Kingdom) university. For purposes of inclusion participants were required to: (1) be 18 years of age or above; (2) consent to participate; (3) understand spoken and written English; and (4) define themselves as 'smokers'. The mean age of the sample was 33.1 years $(S D=11.5)$ and the age range was 18 to 66 years. The sample was 
75.4\% White, 13.1\% Black, 9.5\% Asian, and 2.0\% of mixed ethnicity. Participants’ mean scores on daily cigarette use, the Fagerström Test of Nicotine Dependence (FTND; Heatherton et al., 1991), age of inception of cigarette use and numbers of years using cigarettes were, respectively, 11.2 cigarettes ( $\mathrm{SD}=8.3), 2.9(\mathrm{SD}=2.6), 16.7$ years $(\mathrm{SD}=3.9)$ and 15.4 years $(\mathrm{SD}=10.4)$.

\subsubsection{Materials}

Items representing positive and negative metacognitions about smoking were derived from data collected in an earlier semi-structured interview study (Nikčević \& Spada, 2010), from the authors' clinical experience, and from deductions based on the metacognitive model of psychopathology (Wells, 2009). The items selected as positive metacognitions about smoking concerned the usefulness of smoking in: (1) controlling cognition ("Smoking helps me to focus my mind”); and (2) controlling emotion ("When I get upset smoking comforts me”). The items selected as negative metacognitions about smoking concerned: (1) the uncontrollability of smoking and smoking-related thoughts ("I cannot control my urge to smoke”); and (2) judgments relating to the cognitive interference caused by smoking and smoking-related thoughts ("My thoughts about cigarettes interfere with my functioning”). A total of 48 items were framed in terms of statements to which participants reported the extent of their agreement on a 4-point Likert-type scale ("Do not agree”, “Agree slightly”, “Agree moderately”, “Agree very much”).

\subsubsection{Procedure}

Participants were recruited from a number of work places (a university, a hospital, several schools) using e-mail lists and advertisements. A web link directed the participants to the study website. The first page of the study website explained the purpose of the study as: "To develop a self-report instrument to assess beliefs people hold about smoking”. Participants were then directed, if consenting to participate in the study, to a second page containing basic demographic questions and the self-report instrument. On completion participants were asked to click on the "Submit" button. Once participants had clicked on "Submit”, their data was forwarded to a generic postmaster 
account. This ensured that participants' responses were anonymous. A second submission from the same IP address was not allowed so as to avoid multiple submissions from the same participant.

\subsection{Results}

A principal components method of factor extraction was performed on the scores of the original 48 items. The Scree test suggested a four factor solution (eigenvalues of 16.6, 5.5, 4.0 and 2.2). Items were assessed as indicators of the latent variables using Varimax rotation. The four factors together accounted for $59.9 \%$ of variance. Items which loaded less than 0.4 on any factor were discarded, as were items that loaded on two or more factors. If an item loaded more than 0.4 on one factor, and failed to load onto the other factors, but was within approximately 0.2 of the loading on the first factor, it was also discarded. This procedure was followed in order to exclude items that influenced more than one factor. Only the five items that loaded on each factor were selected to define a brief final version of the self-report instrument. The revised self-report instrument consisted of 20. The factor loadings and communalities of the individual items are presented in Table 1 . Internal consistencies (homogeneity) were determined by computing Cronbach's alpha. This coefficient was 0.92 for factor $1,0.88$ for factor $2,0.85$ for factor 3 , and 0.93 for factor 4 . All items showed correlations above 0.7 on their own factor and above 0.5 with the instrument. Intercorrelation between factors ranged from 0.21 to 0.50 .

Two factors referred to positive metacognitions about the usefulness of smoking in regulating cognition and emotion. We termed these factors 'positive metacognitions about cognitive regulation’ (PM-CR) and ‘positive metacognitions about emotional regulation’ (PM-ER). The third factor referred to the uncontrollability of smoking and smoking-related thoughts. We termed this factor 'negative metacognitions about uncontrollability' (NM-U). The fourth factor referred to the impact and intrusiveness of smoking and smoking-related thoughts on cognitive functioning. We termed this factor 'negative metacognitions about cognitive interference’ (NM-CI). 


\section{Study 2: Confirmation of the Factor Structure and Preliminary Examination of the Predictive and Divergent Validity of the MSQ}

We conducted a second study to confirm the factor structure and test the predictive ability of the MSQ factors. In accordance with the metacognitive model of psychopathology, positive metacognitions about smoking should be involved in the initiation of smoking. Conversely negative metacognitions about smoking may play a role in propagating a smoking episode once it has started (Nikčević \& Spada, 2010). In view of this, we chose daily cigarette use and the FTND as dependent variables to test the predictive validity of the MSQ factors. Divergent validity was tested by comparing the MSQ factors with smoking outcome expectancies, as measured by the Smoking Effects Questionnaire, which have been shown to be significant predictors of smoking (Rohsenow, Abrams, Monti, Colby, Martin \& Niaura, 2003)

\subsection{Method}

\subsubsection{Participants}

A sample of 143 individuals (71 female) participated in the study which was approved by the ethics committee of a London (United Kingdom) university. For purposes of inclusion participants were required to: (1) be 18 years of age or above; (2) consent to participate; (3) understand spoken and written English; and (4) define themselves as 'smokers'. The mean age of the sample was 30.2 years $(S D=10.7)$ and the age range was 18 to 69 years. The sample was 57.3\% White, 35.7\% Asian, 2.0\% Black and $5.0 \%$ of mixed ethnicity. Participants' mean scores on daily cigarette use, the FTND, age of inception of cigarette use and numbers of years using cigarettes were, respectively, 11.5 cigarettes $(\mathrm{SD}=12.2), 3.8(\mathrm{SD}=2.5), 17.7$ years $(\mathrm{SD}=4.7)$ and 12.1 years $(\mathrm{SD}=10.4)$.

\subsubsection{Materials}

The following is a brief description of measures we chose:

The Metacognitions about Smoking Questionnaire (MSQ). The MSQ is the self-report instrument developed in Study 1. 
Smoking Effects Questionnaire (SEQ; Rohsenow, Abrams, Monti, Colby, Martin \& Niaura, 2003). The SEQ is a 33 item self-report instrument to assess smoking outcome expectancies. The SEQ has four factors examining positive smoking outcome expectancies (reduction of negative affect, stimulation, positive social effects and weight control) and three negative smoking outcome expectancies (negative physical effects, negative psychosocial effects and future health concerns). Higher scores denote higher levels of smoking outcome expectancies. The SEQ has been used to report smoking status in smoking populations, and possesses good reliability and validity (Rohsenow, Abrams, Monti, Colby, Martin \& Niaura, 2003).

Fagerström Test of Nicotine Dependence (FTND; Heatherton et al., 1991). The FTND is a brief 6 item self-report instrument to assess nicotine/smoking dependence with scores ranging from 0 to 10 . Higher scores denote higher levels of nicotine dependence, with cut-off points of 3 and 5-6 respectively indicating moderate to high nicotine dependence. The FTND has been widely used to report smoking status in smoking populations, and possesses good reliability and validity (Pomerleau et al., 1994).

\subsubsection{Procedure}

Participants were recruited from email contacts in a viral-like fashion, starting from mailing lists of researchers involved in the study, and directed to the study website. The first page of the study website explained the purpose of the study as: "To determine the utility of a self-report instrument to assess beliefs people hold about smoking”. The rest of the procedure followed that described under section 2.1.3.

\subsection{Results}

\subsubsection{Confirmatory Factor Analysis}

Firstly, a principal components method of factor extraction was performed on the scores to confirm the general factor structure of Study 1. A Scree plot suggested a three factor solution that accounted for $60.9 \%$ of variance. This solution differed from that presented in Study 1 in the fact that the negative metacognitions about smoking items loaded on the same factor. 
Following this, a confirmatory factor analysis (CFA) was conducted on the data obtained from the factor-validation sample using LISREL 8.8 (Jöreskog \& Sörbom, 1996). We compared four models. In Model 1 we defined a single latent variable with all 20 items as indicators. In Model 2 we tested a conceptual two factor solution with positive metacognitions about smoking as a first unique factor and negative metacognitions about smoking as a second unique factor. In Model 3 we defined the three factors as correlated latent variables and the 20 items as congeneric indicators of the latent variables as emerged from the principal components method of factor extraction in Study 2. Finally, in Model 4 we defined a four factor solution as emerged from the exploratory factor analysis in Study 1. We also evaluated the model defining the items as continuous indicators, and using maximum likelihood estimation that assumes multivariate normality of the item scores.

The Root Mean Square Error of Approximation (RMSEA), which indicates the closeness of fit and is sensitive to the mis-specification of the measurement model (the factor loadings), was employed to evaluate the data fit together with the Comparative Fit Index (CFI) and the NonNormed Fit Index (NNFI) as incremental fit indexes. The RMSEA cut-off values close to 0.08 demonstrate adequate fit of the model, whereas between 0 and 0.05 indicate a good fit (Browne \& Cudeck, 1993; Hu \& Bentler, 1999) and values close to or above 0.95 for the CFI and NNFI support an excellent fit.

Model 1 resulted in a RMSEA of 0.24 , a CFI of 0.79 and a NNFI of 0.77 . Model 2 resulted in RMSEA of 0.10, a CFI of 0.93 and a NNFI of 0.92. Model 3 resulted in a RMSEA of 0.08 , a CFI of 0.96 and a NNFI of 0.95 . Model 4 resulted in a RMSEA of 0.07 , a CFI of 0.96 and a NNFI of 0.96. In conclusion Model 4 (the four factor solution) showed the best data fit.

Internal consistencies (homogeneity) were determined by computing Cronbach’s alpha. This coefficient was 0.90 for the total score of the MSQ, 0.93 for PM-CR, 0.76 for PM-ER, 0.81 for NM-U, and 0.86 for NM-CI. Cronbach's alphas were also calculated for all of the items if any single item was removed from the three factors, but this did not result in an improvement in internal consistency. Additionally, Pearson Product-moment correlations between the individual items and 
the relative factor scores were calculated. All of these item-total correlations were above 0.3, suggesting that each item correlated well with the factor score. The inter-correlations between factors were 0.58 between PM-CR and PM-ER, 0.25 between PM-CR and NM-U, 0.34 between PM-CR and NM-CI, 0.38 between PM-ER and NM-U, 0.35 between PM-ER and NM-CI, and 0.74 between NM-U and NM-CI.

\subsubsection{Predictive and Divergent Validity}

Descriptive statistics and inter-correlations for all the self-report instruments are shown in Table 2. An inspection of histograms, and skewness and kurtosis ranges, showed that all scores were normally distributed, with the exception of daily cigarette use, considering both symmetry and peakedness. A square root transformation of the variable daily cigarette use allowed the achievement of acceptable skewness and kurtosis scores.

Two-tailed Pearson correlations showed that all the factors of the MSQ were positively and significantly correlated with daily cigarette use and the FTND. Results also indicated that only positive smoking outcome expectancies (and not negative smoking outcome expectancies) were positively and significantly correlated with daily cigarette use and the FTND. An examination of the inter-correlations between the MSQ and SEQ factors indicated moderate overlap (a maximum correlation of 0.55 between PM-CR and positive smoking outcome expectancies) (see Table 2).

Several steps were taken to assess whether multicollinearity was present in the data. Firstly, the correlation matrix revealed no substantial correlations $(r>.9)$ between the predictor variables. Secondly, an inspection of the ranges of the Tolerance Index and Variance Inflation Factor for all predictor variables supported the absence of multicollinearity. Histograms and normality plots suggested that the residuals were normally distributed. Plots of the regression standardized residuals against the regression standardized predicted values suggested that the assumptions of linearity and homoscedascity were met.

Two hierarchical regression analyses (see Table 3) were run in order to evaluate the contribution of metacognitions about smoking towards daily cigarette use and FTND scores whilst 
controlling for positive smoking outcome expectancies. Negative smoking outcome expectancies were excluded from these analyses because of the absence of significant correlations with the dependent variables. Firstly, a hierarchical regression analysis was run with daily cigarette use entered as the outcome variable and the predictor variables of positive smoking outcome expectancies entered in step 1 and all factors of the MSQ in step 2. Results of this analysis indicated that MSQ factors accounted for $11.1 \%(p<0.001)$ of variance in daily cigarette use above the variance accounted for by positive smoking outcome expectancies $(7.2 \%, p=0.002)$. An inspection of the final equation revealed that negative metacognitions about uncontrollability were the only significant independent predictor of daily cigarette use. A second hierarchical regression analysis was run with the FTND entered as the outcome variable and the predictor variables of positive smoking outcome expectancies entered in step 1 and all factors of the MSQ in step 2. Results of this analysis indicated that MSQ factors accounted for 15.9\% $(p<0.001)$ of variance in FTND scores above the variance accounted for by positive smoking outcome expectancies $(7.2 \%, p<0.001)$. An inspection of the final equation revealed that both negative metacognitions about uncontrollability and negative metacognitions about cognitive interference were the only significant independent predictors of FTND scores. A trend towards significance was also observed for positive metacognitions about cognitive regulation.

\section{Study 3: Temporal Stability of the MSQ}

\subsection{Method}

\subsubsection{Participants}

A sample of 25 individuals ( 9 female) participated in the study which was approved by the ethics committee of a London (United Kingdom) university. For purposes of inclusion participants were required to: (1) be 18 years of age or above; (2) consent to participate; (3) understand spoken and written English; and (4) define themselves as 'smokers'. The mean age of the sample was 32.0 years ( $\mathrm{SD}=12.1$ ) and the age range was 20 to 59 years. The sample was $40.0 \%$ White, $36.0 \%$ Asian, 24.0\% of mixed ethnicity. Participants' mean scores on daily cigarette use, the FTND (Heatherton et 
al., 1991), age of inception of cigarette use and numbers of years using cigarettes were, respectively, 11.9 cigarettes $(\mathrm{SD}=6.7), 3.1(\mathrm{SD}=2.6), 15.6$ years $(\mathrm{SD}=2.4)$ and 16.0 years $(\mathrm{SD}=12.1)$.

\subsubsection{Materials}

In order to assess the temporal stability of MSQ the final version of this self-report instrument was used.

\subsubsection{Procedure}

This was identical to the procedure followed in section 3.1.3. The purpose of the study was: “To examine beliefs about smoking over time”.

\subsection{Results}

An inspection of histograms, and skewness and kurtosis ranges, showed that all scores were normally distributed, considering both symmetry and peakedness. Mean factor scores at testing and retesting were as follows: $12.0(\mathrm{SD}=4.3)$ and $10.8(\mathrm{SD}=4.4)$ for $\mathrm{PM}-\mathrm{CR}, 14.4(\mathrm{SD}=3.3)$ and 12.9 $(\mathrm{SD}=4.2)$ for PM-ER, $10.2(\mathrm{SD}=3.4)$ and $9.8(\mathrm{SD}=3.7)$ for $\mathrm{NM}-\mathrm{U}$, and $7.2(\mathrm{SD}=2.8)$ and 7.5 $(S D=2.6)$ for NM-I. A paired samples t-test indicated that the mean scores for the four factors of the MSQ did not change over the 8-week interval (PM-CR: $\mathrm{t}=1.9, p=0.06$; PM-ER: $\mathrm{t}=1.8, p=0.09$; NM$\mathrm{U}: \mathrm{t}=0.6, p=0.53$; NM-CI: $\mathrm{t}=-0.6, p=0.50$ ) suggesting they possess stable characteristics.

\section{Discussion}

Building on the metacognitive model of self-regulation (Wells \& Matthews, 1994), and on preliminary research on metacognitions in smoking (Nikčević \& Spada, 2008; 2010; Spada, Nikčević, Moneta \& Wells, 2007), we conducted three studies aimed at developing and validating a self-report instrument on metacognitions about smoking.

In Study 1 an exploratory factor analysis suggested a four factor solution comprising of: positive metacognitions about cognitive regulation, positive metacognitions about emotional regulation, negative metacognitions about uncontrollability and negative metacognitions about cognitive interference. Study 2 confirmed the factor structure of the MSQ and revealed that the four identified factors possess adequate internal consistency and low item redundancy. This study also 
showed that all factors of the MSQ are positively and significantly correlated to daily cigarette use and FTND scores (confirming predictive validity) and correlations with SEQ factors are relatively low (indicating divergent validity). This observation was largely confirmed by hierarchical regression analyses which revealed that negative metacognitions about uncontrollability predicted daily cigarette use independently of positive smoking outcome expectancies. In addition both negative metacognitions about uncontrollability and negative metacognitions about cognitive interference predicted FTND scores independently of positive smoking outcome expectancies, with a trend for significance identified for positive metacognitions about cognitive regulation. Finally, Study 3 confirmed the temporal stability of the factors of the MSQ.

These results demonstrate the utility of the MSQ and the potential role of metacognitions in sustaining smoking. It follows that techniques and principles of metacognitive therapy (Wells, 2009) which have been found to be very effective in the treatment of anxiety and mood disorders (Normann, van Emmerik \& Nexhmedin, 2014) could potentially be applied to the discontinuation of smoking. The finding that metacognitions about smoking were a better predictor of smoking behaviour than smoking outcome expectancies is of particular clinical interest. If confirmed, this finding might suggest that cessation of smoking behaviour may depend more on changing metacognitions than on changing cognitive level constructs in treatment.

The findings also align themselves to earlier work undertaken by Spada and colleagues (Spada, Moneta \& Wells, 2007) showing that metacognitions about alcohol use are a stronger predictor of alcohol use than alcohol outcome expectancies. The key similarity between metacognitions about smoking and smoking outcome expectancies is that the positive dimensions of both constructs capture what are essentially motivations for smoking. A crucial difference, however, is that positive smoking outcome expectancies do not explicitly distinguish between cognitive and metacognitive belief domains. This is an important distinction because according to the metacognitive model of psychopathology, and burgeoning research evidence, the key markers of psychopathology are beliefs pertaining to the metacognitive rather than cognitive domain (Wells, 
2000). This may also explain why we identified a trend towards significance for positive metacognitions about cognitive regulation in predicting FTND scores independently of positive smoking outcome expectancies.

Furthermore with respect to the negative dimensions of both scales, whereas negative smoking outcome expectancies mainly measure general negative outcomes arising from smoking, negative metacognitions about smoking tap into the perception of lack of executive control and presumed cognitive interference of smoking and smoking-related thoughts. From a metacognitive standpoint, high scores on negative metacognitions about smoking are the key marker of the perseveration of psychopathology as they may play a key role in propagating negative affect and hence prevent the discontinuation of maladaptive coping behaviour (Nosen \& Woody, 2014; Wells, 2009). The finding that negative metacognitions about smoking were the only significant predictors of both daily cigarette use and FTND scores underscores their possible importance in this role.

The present results are preliminary in nature. The most important limitation is the absence of a longitudinal study that precludes causal inferences. Secondly, the use of snowballing recruitment techniques is subject to biased estimates and is reliant on the skill of the individual conducting the study. Thirdly, the presence of concurrent psychological disorder and exposure to previous treatments aimed at modifying cognition was not assessed. Finally, the presence of concurrent psychological disorder, and the exposure to previous psychological treatments, was not assessed. Clearly future studies are required to further establish the psychometric properties of the MSQ. In particular, it would be necessary to determine the structure and reliability over time and with clinical samples. In addition, studies are required to examine the sensitivity of MSQ to treatment effects and recovery if this self-report instrument is to prove a useful treatment evaluation tool.

Acknowledgements: The authors would like to offer thanks to students See Ann Soo, Elif Syuleymanogly and Guncel Masarogullari who assisted with some of the data collection on this project. 


\section{References}

Browne, M. W. \& Cudeck, R. (1993). Alternative ways of assessing model fit. In K. A. Bollen \& J. S. Long (Eds.), Testing Structural Equation Models (pp. 136-162). Newbury Park, CA: Sage.

Clark, A., Tran, C., Weiss, A., Caselli, G., Nikčević, A. V., \& Spada, M. M. (2012). The Big 5 facets of personality and metacognitions as predictors of level of alcohol consumption in binge drinking university students. Addictive Behaviors, 37, 537-540.

Heatherton, T. F., Kozlowski, L. T., Frecker, R. C. \& Fagerström, K. O. (1991). The Fagerström Test for Nicotine Dependence: A revision of the Fagerström Tolerance Questionnaire. British Journal of Addiction, 86, 1119-1127

Hu, L. \& Bentler, P. M. (1999). Cutoff criteria for fit indices in covariance structure analysis: Conventional versus new alternatives. Structural Equation Modeling, 6, 1-55.

Jöreskog, K. G. \& Sörbom, D. (1996). LISREL 8: User's reference guide. Chicago: Scientific Software International.

Lindberg, A., Fernie, B. A. \& Spada, M. M. (2011). Metacognitions in problem gambling. Journal of Gambling Studies, 27, 73-81.

Nikčević, A. V. \& Spada, M. M. (2008). Metacognitions across the continuum of smoking dependence. Behavioural and Cognitive Psychotherapy, 36, 333-339.

Nikčević, A. V. \& Spada, M. M. (2010). Metacognitions about smoking: a preliminary investigation. Clinical Psychology \& Psychotherapy, 17, 536-542.

Normann, N., van Emmerik, A. P. \& Nexhmedin, M. (2014). The efficacy of metacognitive 
therapy for anxiety and depression: A meta-analytic review. Depression and Anxiety, 31, 402-411.

Nosen, E. \& Woody, S. R. (2014). Acceptance of cravings: How smoking cessation experiences affect craving beliefs. Behaviour Research and Therapy, 59, 71-81.

Pomerleau, O. F., Carton, S. M., Lutzke, M. L., Flessland, K. A. \& Pomerleau, C. S. (1994). Reliability of the Fagerström Tolerance Questionnaire and the Fagerström Test for Nicotine Dependence. Addictive Behaviors, 19, 33-39.

Rohsenow, D. J., Abrams, D. B., Monti, P. M., Colby, S. M., Martin, R. \& Niaura, R. S. (2003). The smoking effects questionnaire for adult populations: Development and psychometric properties. Addictive Behaviors, 28, 1257-1270.

Spada, M. M., Caselli, G. \& Wells, A. (2009). Metacognitions as a predictor of drinking status and level of alcohol use following CBT in problem drinkers: a prospective study. Behaviour Research and Therapy, 47, 882-886.

Spada, M. M., Caselli, G. \& Wells, A. (2013). A triphasic metacognitive formulation of problem drinking. Clinical Psychology \& Psychotherapy, 20, 494-500.

Spada, M. M., Giustina, L., Rolandi, S., Fernie, B. A. \& Caselli, G. (2014). Profiling metacognition in gambling disorder. Behavioural and Cognitive Psychotherapy, published electronically $15^{\text {th }}$ April.

Spada, M. M., Langston, B., Nikčević, A. V. \& Moneta, G. B. (2008). The role of metacognitions in problematic internet use. Computers in Human Behavior, 24, 2325-2335.

Spada, M. M., Moneta, G. B. \& Wells, A. (2007). The relative contribution of metacognitive beliefs and expectancies to drinking behaviour. Alcohol and Alcoholism, 42, 567-574.

Spada, M. M., Nikčević, A. V., Moneta, G. B. \& Wells, A. (2007). Metacognition as a mediator of the relationship between emotion and smoking dependence. Addictive Behaviors, 32, 2120-2129. 
Spada, M. M. \& Wells, A. (2005). Metacognitions, emotion and alcohol use. Clinical Psychology \& Psychotherapy, 12, 150-155.

Spada, M. M. \& Wells, A. (2006). Metacognitions about alcohol use in problem drinkers. Clinical Psychology \& Psychotherapy, 13, 138-143.

Spada, M. M. \& Wells, A. (2008). Metacognitive beliefs about alcohol use: development and validation of two self-report scales. Addictive Behaviors, 33, 515-527.

Spada, M. M. \& Wells, A. (2009). A metacognitive model of problem drinking. Clinical Psychology \& Psychotherapy, 16, 383-393.

Spada, M. M. \& Wells, A. (2010). Metacognitions across the continuum of drinking behaviour. Personality and Individual Differences, 49, 425-429.

Wells, A. (1995). Worry and the incubation of intrusive images following stress. Behaviour Research and Therapy, 33, 579-583.

Wells, A. (2000). Emotional Disorders \& Metacognition: Innovative Cognitive Therapy. Chichester, UK: Wiley.

Wells, A. (2009). Metacognitive Therapy for Anxiety and Depression. New York, USA: Guilford Press.

Wells, A. (2013). Advances in Metacognitive Therapy. International Journal of Cognitive Therapy, 6, 186-201.

Wells, A. \& Matthews, G. (1994). Attention and emotion: A clinical perspective. Hove, UK: Lawrence Erlbaum Associates.

Wells, A. \& Matthews, G. (1996). Modelling cognition in emotional disorder: The S-REF model. Behaviour Research and Therapy, 34, 881-888. 


\section{Tables}

Table 1: Factor Loadings for Individual Items of the MSQ in Study 1 and Study 2 Based on an Exploratory Factor Analyses.

\begin{tabular}{|c|c|c|c|c|c|c|c|c|c|}
\hline & \multicolumn{5}{|c|}{ Study 1} & \multicolumn{4}{|c|}{ Study 2} \\
\hline & F1 & F2 & F3 & F4 & Communality & F1 & F2 & F3 & Communality \\
\hline \multicolumn{10}{|l|}{ Positive Metacognitions about Smoking } \\
\hline \multicolumn{10}{|l|}{ Factor 1: Positive Metacognitions about Cognitive Regulation } \\
\hline (1) Smoking helps me think more clearly & .87 & .07 & .09 & .02 & .77 & .84 & .24 & .08 & .77 \\
\hline (2) Smoking helps me to focus my mind & .87 & .17 & .10 & .17 & .82 & .86 & .16 & .03 & .79 \\
\hline (3) Smoking helps me to order my thoughts & .87 & .08 & .06 & .01 & .76 & .86 & .16 & .13 & .78 \\
\hline (4) Smoking helps me order things in my mind & .81 & .16 & .11 & .16 & .71 & .85 & .22 & .17 & .80 \\
\hline (5) Smoking helps me concentrate & .75 & .28 & .13 & .06 & .67 & .87 & .13 & .15 & .80 \\
\hline \multicolumn{10}{|l|}{ Factor 2: Positive Metacognitions about Emotional Regulation } \\
\hline (1) Smoking helps me to relax when I am agitated & .06 & .81 & .10 & .13 & .69 & .38 & .65 & .04 & .58 \\
\hline (2) When I get stressed smoking calms me down & .06 & .78 & .19 & -.02 & .64 & .26 & .81 & .15 & .74 \\
\hline (3) When I get upset smoking comfort me & .08 & .70 & .21 & -.01 & .54 & .15 & .78 & .20 & .68 \\
\hline (4) Smoking helps me to unwind & .18 & .68 & .04 & .08 & .50 & .24 & .52 & .14 & .35 \\
\hline (5) Smoking distracts me from feeling pressured & .17 & .73 & .06 & .08 & .57 & .48 & .29 & .18 & .35 \\
\hline \multicolumn{10}{|l|}{ Negative Metacognitions about Smoking } \\
\hline \multicolumn{10}{|l|}{ Factor 3: Negative Metacognitions about Uncontrollability } \\
\hline (1) Smoking means I have low will power & .04 & .13 & .78 & .08 & .63 & -.08 & .20 & .59 & .40 \\
\hline (2) It is hard to control my desire for cigarettes & .13 & .25 & .77 & -.01 & .67 & .09 & .31 & .66 & .54 \\
\hline (3) My smoking means that I'm mentally weak & .04 & .12 & .76 & .09 & .60 & -.01 & .12 & .59 & .36 \\
\hline (4) My smoking is uncontrollable & .15 & .15 & .70 & .35 & .66 & .07 & .26 & .74 & .62 \\
\hline (5) I cannot control my urge to smoke & .02 & .20 & .76 & .27 & .69 & .11 & .12 & .76 & .60 \\
\hline \multicolumn{10}{|l|}{ Factor 4: Negative Metacognitions about Cognitive Interference } \\
\hline (1) Thinking so much about smoking interferes with me seeing things clearly & .13 & .03 & .32 & .80 & .77 & .35 & .08 & .63 & .53 \\
\hline (2) I have lost control of my thoughts about smoking & .11 & .02 & .35 & .80 & .78 & .08 & .16 & .73 & .56 \\
\hline (3) My thoughts about smoking are becoming an obsession & .05 & -04 & .36 & .79 & .76 & .15 & .05 & .82 & .70 \\
\hline (4) My preoccupation with cigarettes takes over my life & .19 & -.04 & .36 & .76 & .74 & .16 & -.10 & .75 & .60 \\
\hline (5) My thoughts about the cigarettes interfere with my functioning & .16 & .03 & .31 & .63 & 59 & .26 & -.06 & .76 & .65 \\
\hline
\end{tabular}


Table 2: Means, Standard Deviations, Ranges and Inter-correlations of Variables.

\begin{tabular}{llllllllllll}
\hline & & X & SD & Range & FTND & PM-CR & PM-ER & NM-U & NM-CI & PSOE & NSOE \\
\hline 1. & DCU & 11.4 & 12.3 & $0-120$ & $0.58^{* *}$ & $0.25^{* *}$ & $0.23^{* *}$ & $0.40^{* *}$ & $0.36^{* *}$ & $0.27^{* *}$ & 0.18 \\
2. & FTND & 2.7 & 2.4 & $0-9$ & - & $0.29 * *$ & $0.25^{* *}$ & $0.45^{* *}$ & $0.46^{* *}$ & $0.28^{* *}$ & 0.11 \\
3. & PM-CR & 11.2 & 4.2 & $5-20$ & - & - & $0.61^{* *}$ & $0.27 * *$ & $0.34^{* *}$ & $0.55^{*}$ & 0.04 \\
4. & PM-ER & 13.6 & 3.3 & $6-20$ & - & - & - & $0.39 * *$ & $0.36^{* *}$ & $0.53^{* *}$ & 0.12 \\
5. & NM-U & 9.5 & 3.7 & $5-19$ & - & - & - & - & $0.74 * *$ & $0.48^{* *}$ & $0.48 * *$ \\
6. & NM-CI & 8.1 & 3.4 & $5-18$ & - & - & - & - & - & $0.49^{* *}$ & $0.33^{* *}$ \\
7. & PSE & 44.4 & 11.3 & $21-76$ & - & - & - & - & - & - & $0.31^{* *}$ \\
8. & NSE & 31.8 & 9.5 & $14-56$ & - & - & - & - & - & - & - \\
\hline
\end{tabular}

Note. $n=134$. DCU= Daily Cigarette Use; FTND= Fagerström Test of Nicotine Dependence; PM-CR=Positive Metacognitions Cognitive Regulation; PM-ER=Positive Metacognitions Emotional Regulation; NM-U=Negative Metacognitions Uncontrollability; NM-CI=Negative Metacognitions Cognitive Interference; PSOE=Positive Smoking Outcome Expectancies; NSOE=Negative Smoking Outcome Expectancies.

${ }^{*} p<.05 ;{ }^{* *} p<.01$. 
Table 3: Hierarchical Multiple Linear Regression Statistics with Daily Cigarette Use and FTND as Outcome Variables and Positive Smoking Outcome Expectancies and Metacognitions about Smoking as Predictor Variables.

\begin{tabular}{lcccccc}
\hline & \multicolumn{3}{c}{ DCU } & \multicolumn{3}{c}{ FTND } \\
\hline Step 1 & $\beta$ & $\mathrm{t}$ & $\mathrm{p}$ & $\beta$ & $\mathrm{t}$ & $\mathrm{p}$ \\
PSOE & 0.27 & 3.30 & 0.001 & 0.28 & 3.36 & 0.001 \\
& $r^{2}=0.07$ & & & & $r^{2}=0.08$ & \\
Step 2 & & & & & & \\
PSOE & 0.01 & 0.11 & 0.91 & 0.04 & -0.44 & 0.66 \\
PM-CR & 0.13 & 1.17 & 0.24 & 0.18 & 1.75 & 0.08 \\
PM-ER & 0.00 & 0.02 & 0.98 & 0.03 & -0.30 & 0.76 \\
NM-U & 0.27 & 2.20 & 0.03 & 0.27 & 2.26 & 0.03 \\
NM-CI & 0.11 & 0.91 & 0.36 & 0.23 & 1.99 & 0.05 \\
& $r^{2}=0.18$ & & & $r^{2}=0.26$ & & \\
\hline
\end{tabular}

Note. $n=134$. DCU= Daily Cigarette Use; FTND= Fagerström Test of Nicotine Dependence; PM-CR=Positive Metacognitions Cognitive Regulation; PM-ER=Positive Metacognitions Emotional Regulation; NM-U=Negative Metacognitions Uncontrollability; NM-CI=Negative Metacognitions Cognitive Interference; PSOE=Positive Smoking Outcome Expectancies.

${ }^{*} p<.05 ; * * p<.01$ 


\section{Appendix: Metacognitions about Smoking Questionnaire (MSQ)}

This questionnaire is concerned with beliefs people hold about smoking. Listed below are a number of beliefs that people have expressed. First, try to think about when you smoke. Then, read each item and determine how much you generally agree with it by circling the appropriate number. Please respond to all the items. There are no right or wrong answers.

\begin{tabular}{|c|c|c|c|c|c|}
\hline & $\begin{array}{l}\text { Do not } \\
\text { agree }\end{array}$ & $\begin{array}{l}\text { Agree } \\
\text { slightly }\end{array}$ & $\begin{array}{l}\text { Agree } \\
\text { moderately }\end{array}$ & $\begin{array}{l}\text { Agree very } \\
\text { much }\end{array}$ & Factor \\
\hline 1. Smoking helps me think more clearly & 1 & 2 & 3 & 4 & PM-CR \\
\hline 2. Smoking helps me to relax when I am agitated & 1 & 2 & 3 & 4 & PM-ER \\
\hline 3. Smoking means I have low will power & 1 & 2 & 3 & 4 & $\mathrm{NM}-\mathrm{U}$ \\
\hline $\begin{array}{l}\text { 4. Thinking so much about smoking interferes } \\
\text { with me seeing things clearly }\end{array}$ & 1 & 2 & 3 & 4 & NM-CI \\
\hline 5. Smoking helps me to focus my mind & 1 & 2 & 3 & 4 & PM-CR \\
\hline 6. When I get stressed smoking calms me down & 1 & 2 & 3 & 4 & PM-ER \\
\hline 7. It is hard to control my desire for cigarettes & 1 & 2 & 3 & 4 & $\mathrm{NM}-\mathrm{U}$ \\
\hline $\begin{array}{l}\text { 8. I have lost control of my thoughts about } \\
\text { smoking }\end{array}$ & 1 & 2 & 3 & 4 & NM-CI \\
\hline 9. Smoking helps me to order my thoughts & 1 & 2 & 3 & 4 & PM-CR \\
\hline 10. When I get upset smoking comforts me & 1 & 2 & 3 & 4 & PM-ER \\
\hline 11. My smoking means that I'm mentally weak & 1 & 2 & 3 & 4 & $\mathrm{NM}-\mathrm{U}$ \\
\hline $\begin{array}{l}\text { 12. My thoughts about smoking are becoming an } \\
\text { obsession }\end{array}$ & 1 & 2 & 3 & 4 & NM-CI \\
\hline 13. Smoking helps me order things in my mind & 1 & 2 & 3 & 4 & PM-CR \\
\hline 14. Smoking helps me to unwind & 1 & 2 & 3 & 4 & PM-ER \\
\hline 15. My smoking is uncontrollable & 1 & 2 & 3 & 4 & NM-U \\
\hline $\begin{array}{l}\text { 16. My preoccupation with cigarettes takes over } \\
\text { my life }\end{array}$ & 1 & 2 & 3 & 4 & NM-CI \\
\hline 17. Smoking helps me concentrate & 1 & 2 & 3 & 4 & PM-CR \\
\hline 18. Smoking distracts me from feeling pressured & 1 & 2 & 3 & 4 & PM-ER \\
\hline 19. I cannot control my urge to smoke & 1 & 2 & 3 & 4 & NM-U \\
\hline $\begin{array}{l}\text { 20. My thoughts about cigarettes interfere with } \\
\text { my functioning }\end{array}$ & 1 & 2 & 3 & 4 & NM-CI \\
\hline
\end{tabular}

